\title{
Erratum to: Genetic structure of a QTL hotspot on chromosome 2 in sweet cherry indicates positive selection for favorable haplotypes
}

\author{
Lichun Cai • Roeland E. Voorrips • Eric van de Weg • \\ Cameron Peace • Amy Iezzoni
}

Published online: 22 July 2017

(C) Springer Science+Business Media B.V. 2017

\section{Erratum to: Molecular Breeding. DOI 10.1007/s11032-017-0689-6}

In the original publication, the numbers assigned to each haplotype in Fig. 2 and its expanded version (Supplementary Fig. S2) are not consistent with the numbers used in the article. The haplotype numbers are corrected and the haplotypes are reordered according to these new numbers in the attached revised Fig. 2 and Supplementary Fig. S2.

Fig. 2 Marker allele composition of each haplotype across the five haploblocks for the sweet cherry QTL hotspot on chromosome 2 illustrated using the smallest number of markers needed to differentiate the haplotypes. SSR alleles are recorded as fragment sizes in base pairs. Haplotypes were assigned by the PediHaplotyper software (Voorrips et al. 2016). Haplotypes containing missing marker scores were omitted from the table. The complete marker composition is in Supplementary Fig. S2

Supplementary Fig. S2 Marker allele composition of each haplotype across five haploblocks for the sweet cherry QTL hotspot on chromosome 2. SSR alleles are recorded as fragment sizes in base pairs. The smallest subset of markers needed to differentiate the haplotypes within each haploblock are highlighted in red font. Haplotypes were assigned by the PediHaplotyper software (Voorrips et al., 2016). Haplotypes containing missing marker scores were omitted from the table

The online version of the original article can be found under doi:10.1007/s11032-017-0689-6

L. Cai · A. Iezzoni $(\bowtie)$

Department of Horticulture, Michigan State University, East Lansing, MI 48824, USA

e-mail: iezzoni@msu.edu

R. E. Voorrips · E. van de Weg

Plant Breeding, Wageningen University and Research, 6708

PBWageningen, The Netherlands

C. Peace

Department of Horticulture, Washington State University,

Pullman, WA 99164, USA 


\begin{tabular}{|c|c|c|c|c|c|c|c|c|c|c|c|c|c|c|}
\hline \multirow{2}{*}{ Haploblock } & \multirow{2}{*}{ Marker } & \multirow{2}{*}{$\begin{array}{c}\text { Physical position } \\
(\mathrm{bp}) \\
\end{array}$} & \multirow{3}{*}{$\begin{array}{c}\text { Genetic position } \\
\text { (cM) } \\
\end{array}$} & \multirow{2}{*}{\multicolumn{11}{|c|}{ Haplotypes }} \\
\hline & & & & & & & & & & & & & & \\
\hline \multirow{5}{*}{ HB-B } & & & & 2 & 3 & 4 & 5 & 6 & 7 & 8 & & & & \\
\hline & ss490549037 & $14,319,252$ & 13.5 & $\bar{G}$ & A & G & $\bar{G}$ & A & $\bar{G}$ & $\bar{G}$ & & & & \\
\hline & ss 490549048 & $15,023,181$ & 18.2 & A & $A$ & A & G & G & G & G & & & & \\
\hline & ss490559387 & $15,186,205$ & 19.3 & $\mathrm{C}$ & C & C & $\mathrm{C}$ & C & A & C & & & & \\
\hline & ss490559390 & $15,186,239$ & 19.3 & A & G & G & G & G & A & A & & & & \\
\hline \multirow{5}{*}{$\mathrm{HB}-\mathrm{C}$} & & & & 2 & 3 & 4 & 5 & 6 & 7 & 8 & & & & \\
\hline & ss490547150 & $16,515,942$ & 24.8 & A & A & A & A & G & $A$ & $A$ & & & & \\
\hline & ss490547158 & $17,249,525$ & 28.3 & $A$ & G & A & A & A & G & G & & & & \\
\hline & ss490549121 & $17,503,996$ & 28.6 & G & G & A & G & G & A & G & & & & \\
\hline & ss490549138 & $17,704,790$ & 28.9 & C & A & A & A & C & A & C & & & & \\
\hline \multirow{6}{*}{ HB-D } & & & & 2 & 3 & 4 & 5 & 6 & 7 & 11 & 12 & 14 & & \\
\hline & ss490549172 & $17,862,597$ & 29.0 & C & A & C & C & C & C & $A$ & $A$ & $A$ & & \\
\hline & ss490549192 & $17,940,428$ & 29.0 & G & G & G & G & G & G & G & A & G & & \\
\hline & CPSCT038 & $18,416,459$ & 29.3 & 190 & 204 & 192 & 190 & 192 & 206 & 202 & 192 & 192 & & \\
\hline & ss490556813 & $18,601,465$ & 29.5 & $A$ & A & G & $A$ & A & A & A & A & $A$ & & \\
\hline & ss490556816 & $18,676,414$ & 29.5 & C & C & A & A & A & A & C & A & A & & \\
\hline \multirow{6}{*}{ HB-E } & & & & 2 & 3 & 6 & 7 & 8 & 9 & 10 & 14 & 15 & 16 & 19 \\
\hline & ss490549227 & $18,948,924$ & 29.7 & $A$ & $\bar{G}$ & $A$ & $A$ & A & G & G & $A$ & $A$ & $A$ & $A$ \\
\hline & ss490549238 & $19,068,803$ & 30.8 & G & G & A & G & A & G & A & A & A & A & A \\
\hline & ss490549258 & $19,200,549$ & 31.9 & $A$ & G & G & $A$ & G & A & A & G & A & A & G \\
\hline & ss490549287 & $19,324,328$ & 32.8 & G & A & A & G & A & A & G & G & G & G & G \\
\hline & ss490549331 & $19,470,895$ & 33.4 & G & G & A & $\mathrm{A}$ & G & A & G & A & $\mathrm{A}$ & G & G \\
\hline \multirow{4}{*}{$\mathrm{HB}-\mathrm{F}$} & & & & 2 & 3 & 6 & 7 & 8 & 9 & 10 & 13 & 14 & 16 & 17 \\
\hline & ВРРСТ034 & $20,068,024$ & 40.2 & 255 & 235 & 223 & 237 & 223 & 225 & 223 & 225 & 221 & 255 & 250 \\
\hline & ss 490549443 & $20,126,852$ & 40.3 & $A$ & C & A & $A$ & A & C & C & A & $A$ & A & $A$ \\
\hline & ss490549569 & $20,455,988$ & 42.9 & $\mathrm{C}$ & C & A & $\mathrm{A}$ & $\mathrm{C}$ & C & A & C & $A$ & $A$ & A \\
\hline
\end{tabular}




\begin{tabular}{|c|c|c|c|c|c|c|c|c|c|c|c|c|c|c|}
\hline \multirow[t]{2}{*}{ Haploblock } & Marker & \multirow[t]{2}{*}{$\begin{array}{c}\text { Physical position } \\
\text { (bp) } \\
\end{array}$} & \multirow[t]{2}{*}{$\begin{array}{c}\text { Genetic position } \\
\text { (cM) } \\
\end{array}$} & \multicolumn{11}{|c|}{ Haplotypes } \\
\hline & & & & 2 & 3 & 4 & 5 & 6 & 7 & 8 & & & & \\
\hline \multirow{6}{*}{ HB-B } & ss4900549037 & $14,319,252$ & 13.5 & $\bar{G}$ & $\mathrm{~A}$ & $\mathrm{G}$ & $\bar{G}$ & $A$ & $\bar{G}$ & $\mathrm{G}$ & & & & \\
\hline & ss 490549044 & $14,872,352$ & 17.2 & G & G & G & $G$ & G & $A$ & $G$ & & & & \\
\hline & ss490549048 & $15,023,181$ & 18.2 & A & A & $A$ & G & G & G & G & & & & \\
\hline & ss490549052 & $15,185,727$ & 19.3 & C & A & A & A & A & C & C & & & & \\
\hline & ss490559387 & $15,186,205$ & 19.3 & c & C & C & C & C & A & c & & & & \\
\hline & ss490559390 & $15,186,239$ & 19.3 & A & G & G & G & G & A & A & & & & \\
\hline \multirow{6}{*}{$\mathrm{HB}-\mathrm{C}$} & & & & 2 & 3 & 4 & 5 & 6 & 7 & 8 & & & & \\
\hline & ss490547150 & $16,515,942$ & 24.8 & $A$ & $A$ & $A$ & $A$ & $\bar{G}$ & $A$ & A & & & & \\
\hline & ss490549109 & $17,033,717$ & 26.9 & G & $A$ & G & G & G & G & A & & & & \\
\hline & ss490547158 & $17,249,525$ & 28.3 & $A$ & G & $A$ & $A$ & A & G & G & & & & \\
\hline & ss 490549121 & $17,503,996$ & 28.6 & G & G & $A$ & G & G & $A$ & G & & & & \\
\hline & ss490549138 & $17,704,790$ & 28.9 & C & A & A & A & C & A & C & & & & \\
\hline \multirow{12}{*}{ HB-D } & & & & 2 & 3 & 4 & 5 & 6 & 7 & 11 & 12 & 14 & & \\
\hline & ss490549172 & $17,862,597$ & 29.0 & $\mathrm{C}$ & A & C & $\mathrm{C}$ & C & $\mathrm{C}$ & $A$ & A & $A$ & & \\
\hline & ss490549176 & $17,868,204$ & 29.0 & $A$ & G & G & G & G & G & A & $A$ & G & & \\
\hline & ss490549184 & $17,905,928$ & 29.0 & $A$ & $A$ & $A$ & A & A & A & c & $A$ & C & & \\
\hline & ss490549187 & $17,907,446$ & 29.0 & $\mathrm{C}$ & $C$ & $\mathrm{C}$ & $\mathrm{C}$ & C & C & A & $\mathrm{C}$ & A & & \\
\hline & ss490549192 & $17,940,428$ & 29.0 & G & G & G & G & G & G & G & A & G & & \\
\hline & ss490556795 & $18,223,481$ & 29.2 & G & A & G & G & G & G & G & G & G & & \\
\hline & CPSCT038 & $18,416,459$ & 29.3 & 190 & 204 & 192 & 190 & 192 & 206 & 202 & 192 & 192 & & \\
\hline & ss490556804 & $18,493,620$ & 29.4 & $A$ & $\mathrm{C}$ & C & $\mathrm{C}$ & $\mathrm{C}$ & C & $\mathrm{C}$ & C & C & & \\
\hline & ss490550555 & $18,509,392$ & 29.4 & $\mathrm{C}$ & $A$ & $\mathrm{C}$ & $\mathrm{C}$ & C & A & A & $\mathrm{C}$ & C & & \\
\hline & ss490556813 & $18,601,465$ & 29.5 & A & $A$ & G & A & A & $A$ & A & A & A & & \\
\hline & ss490556816 & $18,676,414$ & 29.5 & $\mathrm{C}$ & C & A & A & A & A & C & A & A & & \\
\hline \multirow{16}{*}{ HB-E } & & & & 2 & 3 & 6 & 7 & 8 & 9 & 10 & 14 & 15 & 16 & 19 \\
\hline & ss490556819 & $18,732,420$ & 29.6 & G & $\bar{G}$ & A & G & A & $A$ & A & A & G & $\bar{G}$ & $A$ \\
\hline & ss490549216 & $18,914,293$ & 29.7 & G & $G$ & $A$ & G & A & G & G & $A$ & G & G & A \\
\hline & ss490549219 & $18,932,477$ & 29.7 & A & $\mathrm{G}$ & G & A & G & G & G & G & A & A & G \\
\hline & ss490549227 & $18,948,924$ & 29.7 & A & $\mathrm{G}$ & A & A & A & G & G & A & A & A & A \\
\hline & ss490549238 & $19,068,803$ & 30.8 & G & $\mathrm{G}$ & A & G & A & G & A & A & A & A & A \\
\hline & ss490549258 & $19,200,549$ & 31.9 & A & $\mathrm{G}$ & G & $A$ & G & $A$ & A & G & A & A & G \\
\hline & ss 490549278 & $19,288,458$ & 31.9 & $A$ & G & G & $A$ & G & G & A & $\mathrm{G}$ & A & A & A \\
\hline & ss490549287 & $19,324,328$ & 32.8 & G & A & A & G & A & A & G & G & G & G & G \\
\hline & ss 490549323 & $19,449,927$ & 33.3 & $\mathrm{G}$ & G & G & $A$ & G & G & G & $\mathrm{G}$ & A & G & G \\
\hline & ss 490549327 & $19,450,637$ & 33.3 & $\mathrm{G}$ & $\mathrm{G}$ & G & $A$ & G & G & G & G & A & G & G \\
\hline & ss490549331 & $19,470,895$ & 33.4 & $\mathrm{G}$ & $G$ & $A$ & A & $\mathrm{G}$ & A & G & A & A & G & G \\
\hline & ss 490549350 & $19,559,740$ & 33.8 & G & G & $A$ & G & G & $A$ & G & G & G & G & G \\
\hline & ss490549371 & $19,675,328$ & 34.2 & A & C & C & $\mathrm{C}$ & C & $\mathrm{C}$ & C & C & C & C & C \\
\hline & ss490549379 & $19,694,934$ & 34.2 & A & G & G & G & G & G & G & G & G & G & G \\
\hline & ss 490549383 & $19,719,211$ & 35.1 & G & $G$ & A & A & A & A & A & $\mathrm{G}$ & A & A & A \\
\hline \multirow{9}{*}{$\mathrm{HB}-\mathrm{F}$} & & & & 2 & 3 & 6 & 7 & 8 & 9 & 10 & 13 & 14 & 16 & 17 \\
\hline & ss490547208 & $19,790,626$ & 37.7 & $A$ & $G$ & $A$ & $A$ & $A$ & G & $A$ & G & $A$ & $A$ & G \\
\hline & ss490549403 & $19,790,626$ & 37.7 & A & $G$ & A & $A$ & A & $\mathrm{G}$ & A & $G$ & A & A & $\mathrm{G}$ \\
\hline & ВРРСТ034 & $20,068,024$ & 40.2 & 255 & 235 & 223 & 237 & 223 & 225 & 223 & 225 & 221 & 255 & 250 \\
\hline & ss 490549443 & $20,126,852$ & 40.3 & $A$ & c & A & $A$ & $A$ & $\mathrm{C}$ & $\mathrm{C}$ & $A$ & $A$ & $A$ & A \\
\hline & ss 490559440 & $20,192,101$ & 40.3 & G & A & G & G & G & G & A & G & G & G & G \\
\hline & ss490549474 & $20,220,616$ & 40.3 & G & A & $\mathrm{G}$ & $G$ & G & G & A & G & G & G & G \\
\hline & ss490549565 & $20,451,849$ & 42.9 & A & A & G & G & A & A & G & A & G & G & G \\
\hline & ss490549569 & $20,455,988$ & 42.9 & c & c & A & A & c & c & A & c & A & A & A \\
\hline
\end{tabular}

